\title{
Verbal Anesthesia: How and When To Use It in Urological Procedures
}

\author{
Steven N Gange and Neil Baum* \\ Tulane Medical School, New Orleans, LA, USA
}

Submission: November 10, 2017; Published: January 22, 2018

*Corresponding author: Neil Baum, Tulane Medical School, New Orleans, LA, USA, Email: doctorwhiz@gmail.com

\begin{abstract}
There is a trend among many urologists to move more procedures from the hospital and ambulatory surgical center into the office. Favorable patient and physician factors are both contributory. The success of this transition requires that patients have a safe and comfortable experience without impacting the quality of outcomes, in the setting of enhanced physician efficiency and satisfaction. This article will discuss the adjunctive role of Verbal Anesthesia in easing the urology patient's in-office procedure experience, with a particular focus on the minimally-invasive UroLift ${ }^{\circledR}$ procedure for BPH. We will define Verbal Anesthesia and review the limited relevant literature, and discuss how to effectively incorporate Verbal Anesthesia into the office setting. Finally we will review our favorable experience with Verbal Anesthesia in facilitating our in-office UroLift $\circledast$ procedures.
\end{abstract}

Introduction: Many diagnostic and therapeutic urological procedures can be performed easily and safely in the office, with limited anesthesia administration. In-office procedures offer many benefits to patients and also to physicians. While adult diagnostic flexible cystoscopy, Botox® injections, vasectomy, and TRUS-biopsy are almost uniformly performed by urologists in-office, other procedures such as InterStim ${ }^{\circledR}$ (stage 1 ), TURBT $<0.5 \mathrm{~cm}$, minimally invasive surgical therapy (MIST) for BPH, and others, are increasingly performed in the office setting, often under strictly local anesthesia. Despite clear advantages, some urologists remain hesitant to expand their in-office procedure armamentarium, while others have successfully managed this transition.

Methods: We have reviewed the literature on use of local anesthesia supplemented by the use of verbal engagement of the patient in the office setting. We have reported on the experience of one of the authors in over 200 cases performed in the office. We have discussed patient selection.

Results: We were able to demonstrate that verbal engagement of the patient can be effective in performing urologic procedures in the outpatient setting.

Conclusion: There is a trend to move procedures from the hospital, to the ambulatory treatment center, to the office setting. Most of these cases can be conducted under local anesthesia with the assistance of verbal anesthesia. We have discussed the technique of using verbal anesthesia in conjunction with local anesthesia.

Keywords: Verbal anesthesia; Urological procedures; In-office patient; Comorbidity; Intraurethral

Abbrevations: MIST: Minimally-Invasive Surgical Therapies; TUNA: Transurethral Needle Ablation; TUMT: Transurethral Microwave Thermotherapy; VA: Verbal Anesthsia

\section{Introduction}

Conducting procedures in the formal operating room environment is a typically straight forward experience for the physician, however sacrifices must also be made. First, being away from the office to conduct surgical cases seriously impairs physician efficiency. Even when a day of OR cases begins and flows as scheduled, turnover time in the hospital or ASC can cause gaps of 30-60 minutes between cases, time often spent drinking coffee in the Doctor's Lounge without any access to the office EMR (in which case messages and patient results that inevitably accumulate must be dealt with at a later time, further compounding time lost). This inefficiency, affecting almost all surgeons, creates loss of productivity (both in terms of the physician's remuneration, but also freedom to accomplish both in-office and leisure activities). On the contrary, when working in the office, the doctor has full access to the "to-do list" of the EMR, enjoys greater control of the schedule, and can potentially reduce the lost time between cases. Although certain office procedures require a long set-up or local anesthetic dwell time, the surgeon has the possibility of seeing other patients or doing chart work during his/her down time, maximizing overall productivity. Most procedures also offer the physician clear economic advantages when performed in-office. 
Patients also appreciate some unique benefits of inoffice procedures. The average patient has already become comfortable in the office and with the office staff, and perceives large "facilities" as somehow threatening (e.g., believing these are where "big and risky surgeries" are performed, and even "hospitals are where people die"). Thus, patients are generally more reticent to accept procedures at hospitals or ASCs if an in-office option exists for them, and scheduling barriers for in-office procedures are fewer. There is also measurable stress related for the prep for "facility cases". Typically, for procedures performed in the office there is no required fasting period, and no needle sticks for labs or IV placement. Additionally, offices don't typically require a separate anesthesia consent or Advance Directives, and an established in-office patient doesn't have to regurgitate a health history or insurance policy details prior to the procedure. Having less paperwork to complete is definitely appreciated by patients. Also, in general, from a financial standpoint, patient co-pays and out of pocket costs are lower for office procedures. And since many urological procedures can be done under strictly local anesthesia (without sedation) this allows patients with significant comorbidities to complete an in-office procedure with much lower risk. Just as the doctor's time is more efficiently spent when in-office, patients who might spend the better part of a day at a facility even for a short OR case are often able to spend less than an hour completing and recovering from a straightforward office procedure. Importantly, safety concerns for elderly patients who could experience a fall and even fracture after receiving sedation are much lower when a procedure is performed in-office under local anesthesia, and this is uniformly appreciated by physicians, patients, and their caregivers. Finally, as opposed to surgical facility requirements, many patients also enjoy the freedom of driving themselves home without an escort after a straight forward office procedure.

\section{Selecting Patients for In-Office Procedures}

Obviously, and foremost, the proposed procedure must be proven tolerable before it is offered in an awake patient office setting. Ideally, evidence comparative experience should exist demonstrating equivalent efficacy for a procedure performed in-office or in an OR. And, critically, some form of assessment, whether subjective or objective, of patient anxiety or pain tolerance should be performed in anticipation of offering an inoffice procedure. This assessment begins with the initial patient interview and exam. We suggest paying attention to potential "red flags" including unusual levels of conversational anxiety, a history of treatment for an anxiety disorder or chronic pain, known drug or alcohol abuse, numerous narcotic "allergies", and/or an unexpected degree of discomfort associated with scrotal or digital rectal exam, as these patients will have difficulty tolerating in-office procedures. Beyond that initial encounter, a further assessment of tolerability can be made if a screening flexible cystoscopy and/or trans-rectal ultrasound are part of the workup. If the patient is intolerant to flexible cystoscopy (even with intraurethral lidocaine) or TRUS, then it is unlikely that he will tolerate an in-office procedure without some form of sedation-in the rare cases of extreme discomfort during the assessment we will elect not to offer in-office procedures to these patients at all. The Prostatic Urethral Lift (PUL, UroLift ${ }^{\circledR}$ implant, NeoTract, Pleasanton, CA): A model for in-office procedures with limited anesthesia.

Most urologists are comfortably familiar with several traditional office-based procedures, including vasectomy, flexible cystoscopy, and TRUS-guided prostate biopsy. Historically, minimally-invasive surgical therapies (MIST) for BPH such as transurethral needle ablation (TUNA) and transurethral microwave thermotherapy (TUMT) were performed in urology offices but were often perceived by patients as relatively uncomfortable; this, combined with published data demonstrating a lack of proven durability, has resulted in fewer of these procedures performed in the US over the past few years. Of the more than 12 million men treated annually in the U.S. for $\mathrm{BPH}$, only $133,000(<1 \%)$ will have a prostate procedure or prostate surgery [1]. In the case of the more recently available MIST for BPH, UroLift ${ }^{\circledR}$, published clinical data entirely supports a tolerable local anesthetic approach, despite deployment of the implants through a 20 French rigid cystoscopy sheath [2]. In fact, the randomized, controlled, pivotal L.I.F.T. trial that launched in North America in February 2011 and led to FDA-approval was conducted entirely under local (almost exclusively topical) anesthesia, with minimal oral sedation (only 4 of 140 men received prostate blocks), and oral diazepam $10 \mathrm{mg}$ was given 30 minutes prior to the procedure); all subjects were administered both intravesical and intraurethral lidocaine. Prior to this trial all UroLift $®$ procedures had been performed in the operating room under general anesthesia.3 Objective outcome measures among L.I.F.T. subjects compares favorably to those whose procedures were performed under general anesthesia, and no L.I.F.T. study procedure was aborted for subject discomfort. The subsequent LOCAL trial assessed the overall patient in-office UroLift ${ }^{\circledR}$ experience, finding that the mean VAS pain score during UroLift under local with oral sedation was not much different from that reported during the screening office flexible cystoscopy (5 vs 3 on the VAS pain score) [2].

One of us (SG), who performed the first UroLift ${ }^{\circledR}$ under local in the world (as a L.I.F.T. principal investigator), has now completed over 200 cases all of which have been performed inoffice, under local anesthesia. Meanwhile, his specific anesthetic technique has been simplified over time. Because of patient safety concerns, oral sedation is now rarely and very selectively offered ( $<10 \%$ of cases), The administration of intravesical lidocaine has been aborted because it requires the uncomfortable passage of a catheter and appears to add little to UroLift ${ }^{\circledR}$ tolerability. The current in-office UroLift@ anesthesia protocol now typically relies solely upon delivery of $20 \mathrm{cc}$ of chilled $2 \%$ lidocaine jelly, with penile clamping for a strict 20 -minute period prior to scope insertion. No procedure using this in-office local anesthesia practice has ever been aborted for patient discomfort [3]. 
The two points of predictable discomfort during UroLift $®$ always seem to include the repeated antegrade passage of the rigid scope through the bladder neck (which may require some specific patient coaching), and the urgency associated with a full bladder (most easily manageable by minimizing irrigant flow); modifying the anesthetic technique has not made inoffice UroLift ${ }^{\circledR}$ any less tolerable. In fact, patient cooperation is maximized under strictly topical anesthesia, which seems to make the procedure flow more smoothly. Also, we always perform UroLift $\AA$ on the other side of a patient blinding screen, minimize cross-talk and loud exchanges between staff, and have music playing in the procedure room, all of which we have subjectively determined to be useful in minimizing patient anxiety. This refined methodology has been taught and successfully implemented among UroLift ${ }^{\circledR}$ users across the US, and it has become apparent that, once the urologist and staff are trained and comfortable, a surgeon with reasonable endoscopic skill, quiet hands, and a calm demeanor can reproducibly offer UroLift ${ }^{\circledR}$ to patients under local, even strictly topical, anesthesia (often without oral or IV sedation) with acceptable tolerability and excellent outcomes. In addition to the previously described patient-centered advantages in this setting, awake and alert patients appear to pass post-procedure voiding trials readily (resulting in fewer than $10 \%$ requiring catheters in our UroLift $\AA$ practice). The vast majority of these men will have spent less than an hour of total time in the office, and will be able to comfortably and safely drive themselves home. But it is the addition of Verbal Anesthesia, described below, employed throughout the L.I.F.T. trial and diligently and continually used in our clinical practice that we deem indispensable to in-office, local anesthetic UroLift $®$ patient tolerability [4].

Alternative anesthetic approaches for UroLift $®$ implantation include virtually any technique available. In fact, one of us (NB) has recently pioneered the integration of inhalational Nitrous Oxide (N20) delivery into his UroLift $®$ local anesthetic protocol, and has witnessed remarkable patient acceptance and tolerability. Similar to a strictly topical anesthetic approach, the rapid washout of $\mathrm{N} 20$ facilitates maintenance of baseline neurological parameters with minimal safety risk and reliability of trial passage. N2O patients recover quickly and can drive themselves home. Unfortunately, on the downside, there are some significant start-up costs, physician and staff training issues (and even potential for staff abuse), and special ventilation requirements. Finally, the supply expense for N2O administered per case may be difficult to recoup. Therefore, this is an out of pocket expense to the patient of approximately $\$ 50$. For these reasons, at this time widespread adoption of this creative anesthetic solution by urologists may be limited.

\section{Verbal Anesthesia (aka "Vocal Local")}

Verbal Anesthesia (VA) is the art of conversational distraction associated with measures to ensure a calming environment. It is commonly but haphazardly used by in-office surgeons, and is poorly described in the literature. Generally speaking, wellfocused VA draws a patient's attention away from negative stimuli, thereby reducing pain, anxiety and stress. In so doing, VA encourages a procedural environment that helps to maintain relaxation, well-being, and comfort.

\section{Applied technique of Verbal Anesthesia and enhancing the UroLift $\circledast$ patient experience}

Good VA begins with clear pre-operative communication. We believe it is important to set patient expectations at the time of scheduling an office procedure. On the day of UroLift $®$ implantation it is imperative to inform patients of the predictable sensations and sounds involved in administration of local anesthetic and UroLift $\AA$ deployment. A medical assistant (MA) in is assigned the role of "verbal anesthetist" for the case, and sets the tone when rooming the patient and administering intraurethral lidocaine. Upon entering the procedure room the patient will note that the room temperature is made comfortable and soothing music is playing; he then undresses privately from the waist down and lays supine on the procedure table under a drape. After re-entering the room, the MA sets up the blinding screen, removes the patient's drape, preps with warm soap, and describes the sensation of fullness and the cold temperature of the jelly prior to its administration. The conversation is then deliberately shifted to something entirely unrelated (e.g., weather, family, hobbies, etc.) as the local anesthesia is administered and the penile clamp is applied, and this conversation continues through the procedure. Next, during the 20-minute dwell period the urologist will enter the room and describe a bit more about the procedural experience (including the cold water dribbling over the scrotum during cystoscopy, and the sharp and ratcheting noises made with each implant deployment); at this point patient's level of anxiety is carefully assessed, and of course any remaining questions will be answered. The hope of this standardized approach is to reduce the likelihood of surprises to the patient during the procedure. It is our experience that a well-informed and artfully distracted patient tolerates UroLift $\AA$ better and has less anxiety and discomfort. As the procedure begins, there is additionally a need for specific coaching comments from the urologist. When such encouragement is offered, we are very selective with our choice of phrases used, opting for phrases like "you will feel some cold water", "breathe slowly and easily", "try to wiggle your fingers and toes", "here come those noises I told you about", and "let me know when your bladder is full", while specifically avoiding such stress-inducing phrases as "okay, I'm going to insert the scope now", "this is going to hurt a little", "I'm going to fire the gun/device", or "hold your breath", and we almost never utter the word "pain"-it seems intuitive to us that certain words and phrases actually increase a patient's anxiety. Meanwhile, the more the verbal anesthetist can personalize the conversation, the more likely that the patient can be effectively distracted throughout the procedure, and the fewer such physician coaching comments will be required. We believe as a result of our attention to these principles that when 
offered the opportunity to rate their overall discomfort with the procedure in this setting on a 1-10 scale our patients typically respond in the 4-5 range, very seldom does a patient complain about the discomfort of his UroLift $®$ experience.

\section{Discussion}

An individual's perception of pain is not a predictable constant but is variable, complicated by subjective behavioral issues that make it difficult to manage with a cookbook approach. Pain perception involves many somatic and autonomic system signals such as physical, nervous, endocrine, and psychosocial factors, and often complex interactions between these. Various methods have been employed in the hopes of reducing pain perception in awake patients.

For millennia, the only available anesthetic was verbal, occasionally supplemented by alcohol ingestion. As such elective surgery was rare. Finally, in the 1840 s, when it was demonstrated that ether, nitrous oxide, and chloroform could prevent the pain, an increase in the frequency of surgery followed. Nonetheless, in the absence of trained anesthetists and without the ability to adequately monitor vital signs, the dangers of anesthesia from caused many surgeons to shy away from anesthesia. In fact from 1853 to 1862, 32\% of all amputations at Pennsylvania Hospital were performed without any anesthesia whatsoever. For a variety of socioeconomic and even superstitious factors the prevailing view of surgeons in the 19th century was against the use of anesthetic in surgery [5]. Then, and pivotally, in 1884, a Viennese scientist Carl Koller demonstrated the efficacy of topical cocaine in ocular anesthesia, allowing for pain management without the lethal side effects of inhalational anesthesia [6]. Urologists soon successfully experimented with intraurethral cocaine, creating a new paradigm in the specialty where treatment did not depend on suffering, and a revolution in the practice of urological surgery ensued [7]; unfortunately, even topical cocaine use was associated with adverse events and addiction, limitations widely recognized by the early 20 th century. By the 1920s, other less-risky intraurethral options emerged including Novocain, procaine, apothesine, and alypin [8]. Xylocaine, an even safer alternative, emerged and a study published in 1953 found it to be an effective method of local anesthesia during multiple types of cystoscopic cases, avoiding the need for general anesthesia [9]. By the 1990s, $2 \%$ lidocaine gel became widely available. Since that time, numerous studies have investigated the volume, temperature, amount of preprocedural intraurethral dwell time, and cost-effectiveness of intraurethral anesthetic. No well-performed large study has convincingly demonstrated the efficacy or lack thereof of intraurethral lidocaine over lubricating jelly alone for flexible cystoscopy. Nonetheless, many urological surgeons who perform cystoscopic procedures in-office, offer their patients topical anesthesia. And chilled intraurethral lidocaine gel has emerged, at least in a single study, as more effective at achieving analgesia than room-temperature or warmed lidocaine gel [10].
Given the numerous options in the modern era, some physicians simply opt to quiet the patient and thereby avoid the stress of dealing with patient distress and discomfort. To this end, typically, oral benzodiazepines are chosen for their apparent ease of use. In our early in-office UroLift experience we also added an oral narcotic for maximal "comfort", and not surprisingly observed dense sedation in some of our patients; an important, related, and recent FDA Drug Safety Communication announced a Boxed Warning for the combination of oral narcotics and benzidiazepines, related to resultant potential respiratory and CNS depression, including death [11]. Other surgeons use formal intravenous conscious sedation (with benzodiazepines or even propofol), accepting the necessary costs including hiring an R.N. assistant, cardiopulmonary monitoring requirements, and even ACLS certification and supplemental oxygen and crash cart availability. Nevertheless, even when performed conscientiously, patient safety issues arise in these settings that do not exist when using strict local anesthesia. Often underappreciated is the lingering effect of such medications, particularly in the elderly: not only are these patients at higher risk in the office itself, but falls and fractures are more likely during transfers in and out of the vehicle or even once "safely" home.

Some in the anesthesia literature have advocated the use of hypnosis in conjunction with verbal techniques, noting limitations in widespread use related to requirements for special training and necessary time consumed [12]. In addition, many in-office procedures seem almost randomly accompanied by music. While this may be a decision based on expressed personal preferences of the physician, patient, or even staff members, there is in fact evidence that music objectively improves the procedural experience for the awake patient. In the urological literature, randomized trials have demonstrated that music enhances the tolerability for male patients undergoing either flexible [13], or rigid cystoscopy [14], and transrectal ultrasound-guided prostate biopsy [15]. Music appears to improve psychological and physiological parameters; it also offers anxiolytic effects and has been successfully applied to ameliorate stressful interventions [16]. Also, studies have shown the importance of education prior to prostate biopsy in reducing procedural and post-procedural anxiety and discomfort [17]; it appears that anxiety increases pain perception through the activation of the adrenergic response and it is proposed that education can reduce this anxiety [17].

A PubMed literature search for "Verbal Anesthesia" yields very little. One "step-by-step" guide emphasizes the importance of saying the "right thing at the right time"; we have taken it a step further, believing it is equally important not to say the wrong thing out of concern for evoking anxiety. Although VA is poorly defined and studied, its use seems not to be on the radar for many procedures oriented doctors. Settings involving awake patients where, at least anecdotally, VA has been proven as essential in achieving patient tolerability includes dentists and oral surgeons doing everything from tooth extractions to 
dental implants, dermatologists performing Mohs microsurgery, plastic and reconstructive surgeons using in-office liposuction for fat remodeling and employing LASER techniques, ENTs opening sinuses with balloons, ophthalmologists performing LASIK surgery, gynecologists conducting hysteroscopy and uterine ablations, and urologists doing vasectomies and prostate biopsies, just to name a few $[18,19]$. Despite a lack of formal training, most physicians including urologists have begun to adopted VA to some limited degree.

\section{Bottom Line on Verbal Anesthesia}

VA is a simple technique of saying the right thing at the right time, while avoiding words and phrases that evoke anxiety, and thus creating a relaxed procedural environment by focusing a patient's attention away from something anxiety-producing and onto something more familiar. Certainly a patient needs to be well-informed and the procedure room environment needs to be calm, and music should be playing in the background. Then by properly and consistently employing VA, the in-office procedure team can assist a properly selected patient through almost any office procedure, and enhance the overall patient treatment day experience, without adding safety concerns associated with adjunctive medications. Based now on more than five years of experience, we assert VA is an indispensable adjunct to in-office UroLift ${ }^{\circledR}$ implantation, particularly when relying on strictly topical local anesthesia. This straightforward approach has been introduced into and validated within numerous US urology practices, and clearly yields direct physician and patient benefits [19]. In the end, we believe that VA be an addition to local anesthetic procedures: it's inexpensive and easy to learn, there are no allergic reactions, and a patient can't overdose on VA!

\section{References}

1. Egan KB (2016) The Epidemiology of Benign Prostatic Hyperplasia Associated with Lower Urinary Tract Symptoms: Prevalence and Incident Rates. Urol Clin North Am 43 (3):289-297.

2. Shore N, Freedman S, Gange S, Moseley W, Heron S, et al. (2014) Prospective multi-center study elucidating patient experience after prostatic urethral lift. Can J Urol 21(1):7094-7101.

3. Personal experience of author, Dr. Steve Gange.

4. Roehrborn CG, Gange SN, Shore ND, Giddens JL, Bolton DM, et al. (2013) The prostatic urethral lift for the treatment of lower urinary tract symptoms associated with prostate enlargement due to benign prostatic hyperplasia: The L.I.F.T. Study. J Urology 190(6): 2161-2167.

5. Pernick MS (1983) A calculus of suffering, pain, professionalism, and anesthesia in nineteenth-century America. The Hastings Center Report. 13(2): 26-36.
6. Spillane J (2000) Cocaine from medical marvel to modern menace in the United States, 1884-1920. In: Baltimore (Eds.), Johns Hopkins, Myrland.

7. Gordetsky J, Bendana E, O’Brien J, Rabinowitz R (2011) (Almost) painless surgery: A historical review of the evolution of intraurethral anesthesia in urology. Urology 77(1): 12-16.

8. Russell A (1923) The use and abuse of local anesthetics. J Urol 10: 503518.

9. Persky L, Davis HS (1953) Xylocaine as a topical anesthetic in urology. J Urol 70(3): 552-554

10. Thompson TJ, Thompson N, O'Brien A, Young MR, McCleane G (1999) To determine whether the temperature of $2 \%$ lignocaine gel affects the initial discomfort which may be associated with its instillation into the male urethra. BJU International 84(9): 1035-1037.

11. FDA (2016) FDA Drug Safety Communication: FDA warns about serious risks and death when combining opioid pain or cough medicines with benzodiazepines; requires its strongest warning. U S Food and Drug Administration.

12. Stone AB, Sheinberg R, Bertram A, Seymour AR (2016) Are Anesthesia Providers Ready for Hypnosis? Anesthesia Providers' Attitudes Toward Hypnotherapy. Am J Clin Hypnosis 58(4): 411-418.

13. Zhang Z, Wang X, Xu C, Zhang C, Cao Z, et al. (2014) Music reduces panic: An initial study of listening to preferred music improves male patient discomfort and anxiety during flexible cystoscopy. J Endourol 28(6): 739-744.

14. Yeo JK, Cho DY, Oh MM, Park SS, Park MG (2013) Listening to music during cystoscopy decreases anxiety, pain, and dissatisfaction in patients: a pilot randomized controlled trial. J Endourol 27(4): 459462.

15. Cho SW, Choi HJ (2016) Effect of music on reducing anxiety for patients undergoing transrectal ultrasound-guided prostate biopsies: Randomized prospective trial. Urol J 13(2): 2612-2614.

16. Lee DW, Chan KW, Poon CM, Ko CW, Chan KH, et al. (2002) Relaxation music decreases the dose of patient-controlled sedation during colonoscopy: A prospective randomized controlled trial. Gastrointest Endosc 55(1): 33-36.

17. Chiu LP, Tung HH, Lin KC (2016) Effectiveness of stress management in patients undergoing transrectal ultrasound-guided biopsy of the prostate. Patient Prefer Adherence 10: 147-152.

18. Wade J, Rosario DJ, Howson J, Avery KN, Salter CE, et al. (2015) Role of information in preparing men for transrectal ultrasound-guided prostate biopsy: A qualitative study embedded in the ProtecT trial. BMC Health Serv Res 15: 80-93.

19. LVC(2017) Step-by-step-guide-to-verbal-anesthesia. LondonVisionClinic.com 2009. London Vision Clinic, London, UK. 
Your next submission with Juniper Publishers will reach you the below assets

- Quality Editorial service

- Swift Peer Review

- Reprints availability

- E-prints Service

- Manuscript Podcast for convenient understanding

- Global attainment for your research

- Manuscript accessibility in different formats

( Pdf, E-pub, Full Text, Audio)

- Unceasing customer service

Track the below URL for one-step submission https://juniperpublishers.com/online-submission.php 